\title{
Spasticity and white matter abnormalities in adult phenylketonuria
}

\author{
P A McCombe, D B McLaughlin, J B Chalk, N N Brown, J J McGill, M P Pender
}

\begin{abstract}
A 19 year old male with phenylketonuria (PKU) developed a spastic paparesis 8 months after stopping his restricted phenylalanine diet. CT and MRI showed abnormalities of the deep cerebral white matter, and visual evoked response latencies were prolonged. The spasticity gradually improved over several months after resuming the PKU diet. A repeat MRI scan was unchanged. His brother also had PKU and ceased dietary restrictions, but his only neurological abnormality was a slight increase in the deep tendon reflexes of the lower limbs. CT and MRI of his brain was normal. DNA analysis showed that both brothers were homozygous for the same PKU mutation. These patients demonstrate that reversible neurological signs may develop in patients with classic PKU after ceasing dietary restrictions and that these may be associated with abnormalities seen on neuroimaging.
\end{abstract}

Phenylketonuria (PKU) is an autosomal recessive disorder characterised by hyperphenylalaninaemia due to deficiency of hepatic phenylalanine hydroxylase. If untreated, phenylketonuria leads to mental retardation and other neurological abnormalities. ${ }^{12}$ Treatment of PKU by restriction of dietary phenylalanine prevents the development of mental retardation but cessation of the restricted diet may lead to intellectual or neurological deterioration. $^{34}$ We describe a patient with an unusual type of neurological deterioration which occurred after cessation of dietary therapy and improved after re-institution of the diet.

\section{Case histories}

Patient 1

This 19 year old male PKU patient presented with a 6 month history of progressive stiffness, difficulty with walking, difficulty rising from a squatting position, weakness of the upper limbs and slowness of speech. His family stated that he had become irritable. Hyperphenylalaninaemia had been detected by a newborn screening programme. He had been treated with a restricted phenylalanine diet and with oral vitamin supplements from 16 days of age until 8 months before presentation. His serum phenylalanine levels were always above the normal range even while on the diet. From the age of 12-19 years, the serum phenylaline levels ranged from a mean of $893->1200$ $\mu \mathrm{mol} / \mathrm{l}$ (normal < 120). He developed normally and had attended school until the age of 16 when he was falling behind academically. His IQ (Wechsler AIS) was 88 at age 16 years. Before the development of neurological problems he had worked on the family farm.

On examination he was fair-haired, had pale skin and was irritable and uncooperative. Speech was slow and the jaw jerk was brisk. In the upper limbs there was increased tone and in the lower limbs there was spasticity. There was mild pyramidal weakness of all limbs. The deep tendon reflexes were markedly increased and the plantar responses were extensor. The remainder of the physical examination was normal.

On admission, the serum phenylalanine concentration was $1900 \mu \mathrm{mol} / 1$ (normal $<120$ ). The serum vitamin B12 level was 141 ng/l (normal, 145-700). The red cell folate level was $157 \mu \mathrm{g} / 1$ (normal, 150-750). The cerebrospinal fluid (CSF) protein level was $300 \mathrm{mg} / \mathrm{l}$ (normal < 400). Porphyrin levels and serum levels of copper, caeruloplasmin and selenium were normal. Syphilis and human immunodeficiency virus studies were negative. Levels of aryl sulphatase A, beta-galactocerebrosidase, beta-glucocerebrosidase, sphingomyelinase, beta-galactosidase, $\mathrm{N}$-acetylbeta-hexosaminidase, alpha-mannosidase, alpha-fucosidase and beta-glucuronidase were normal. There were no metachromatic granules in the urine. The C26:C22 and C24:C22 very long chain fatty acid ratios were normal. The serum phytanic acid concentrations were normal. An electroencephalogram showed a mild nonspecific excess of slow activity. Visual evoked potential latencies were $121 \mathrm{~ms}$ and $123 \mathrm{~ms}$ from the left and right eyes respectively (normal $<117 \mathrm{~ms}$ ). Nerve conduction studies of the right median and ulnar nerves and left peroneal, tibial and sural nerves were normal. A cervical myelogram was normal. MRI (figure) showed symmetrical abnormalities of the deep posterior cerebral white matter. Analysis of DNA with probes for the known PKU haplotypes ${ }^{5}$ showed that the patient was homozygous for the type 2 haplotype which confirms the diagnosis of classical PKU.

The patient was re-started on a restricted protein diet and given PK Aid III (Scientific Hospital Supplies) dietary supplements. His irritability settled quickly and over the following 2 months he showed gradual neurological improvement with reduction of spasticity. On review after 2 months he was reinvestigated, 

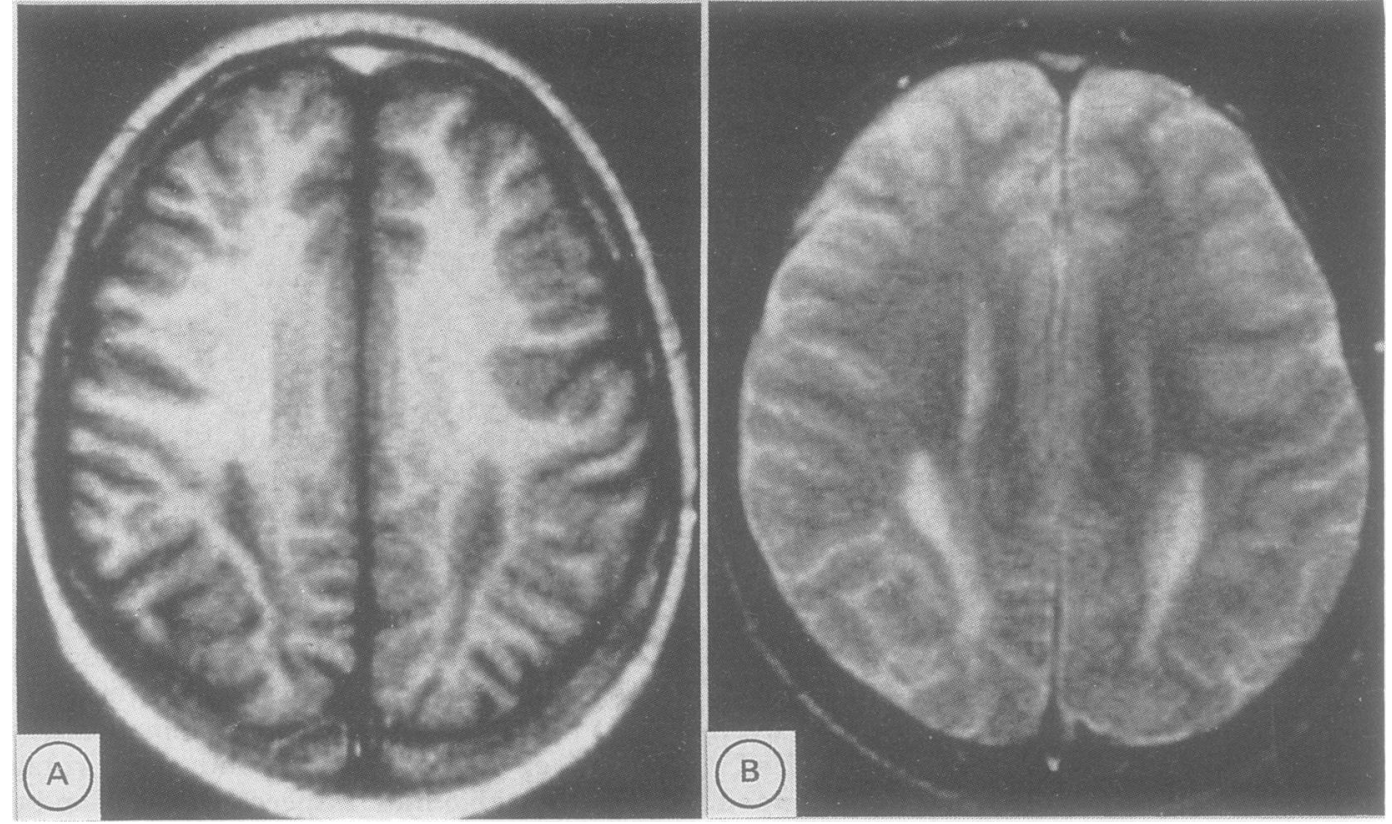

Figure (A) $T 1$ weighted MRI scan (TE, 30;TR, 1500) of patient 1 showing regions of reduced signal intensity in the deep posterior cerebral white matter; (B) T2 weighted MRI scan (TE, 85; TR, 3000) showing areas of increased signal intensity in the deep posterior cerebral white matter.

and the serum phenylalanine concentration was $1960 \mu \mathrm{mol} / \mathrm{l}$ and CSF phenylalanine concentration was $930 \mu \mathrm{mol} / \mathrm{l}$. At this time the serum tyrosine concentration was $29 \mathrm{~mol} / \mathrm{l}$ (normal, 50-90), the CSF tyrosine concentration was $14 \mu \mathrm{mol} / 1$ (normal 2-10) and the CSF glutamine concentration was $740 \mu \mathrm{mol} / 1$ (normal, 150-550). The serum B12 concentration was again measured and was $167 \mathrm{ng} / 1$ (normal 145-700). A Schilling's test was normal. He was started on regular intramuscular vitamin B12 therapy. Over the succeeding months his neurological condition continued to improve. By 6 months after presentation his spasticity had almost totally disappeared. However, the serum phenylalanine concentrations continued to be elevated at a concentration of 1760 $2410 \mu \mathrm{mol} / 1$. A repeat MRI scan was unchanged.

Patient 2

This 21 year old male is the brother of patient 1. He had also been diagnosed in the neonatal period as having PKU and was treated from 21 days of age. He had developed normally and attended school until the age of 14 years. His IQ (Wechsler AIS) was 95 at the age of 18 years. He remained on a restricted diet until aged 18 although his serum phenylalanine concentrations were elevated even while on the diet. From age 12-17 years, the mean serum phenylalanine levels ranged from $310->$ $1200 \mu \mathrm{mol} / 1$ (normal < 120). He then started a normal diet without any intellectual or neurological deterioration.

On examination he had fair hair and pale skin. General physical examination was normal. The deep tendon reflexes of the lower limbs were slightly increased but the remainder of the neurological examination was normal. Serum phenylalanine was $1650 \mu \mathrm{mol} / 1$ (normal $<120$ ). Serum vitamin B12 concentration was
$184 \mathrm{ng} / 1$ (normal 145-700) and the red cell folate level was $186 \mu \mathrm{g} / 1$ (normal 150-750). An electroencephalogram was normal. Visual evoked response latencies were $122 \mathrm{~ms}$ and $104 \mathrm{~ms}$ from the left and right eyes respectively. CT and MRI brain scans were normal. DNA analysis showed the patient was homozygous for the PKU haplotype 2 .

\section{Discussion}

In both patients phenylketonuria had been diagnosed in a newborn screening programme. Both were shown by DNA analysis to be homozygous for haplotype 2 , which confirms the diagnosis of PKU, of the severe classical type. ${ }^{6}$ They had been treated with restricted phenylalanine diets and had developed normally with minimal mental retardation. Patient 1 ceased the PKU diet at the age of 18 , and developed severe spasticity and mild weakness. His serum phenylalanine levels were elevated and CT and MRI brain scans and visual evoked responses were abnormal. When he resumed the restricted diet his spasticity improved. He was extensively investigated for other conditions, including leukodystrophy, and the only abnormal finding was a borderline low vitamin B12 concentration. Patient 2 who had ceased the diet and who also had elevated serum phenylalanine had no evidence of spasticity although the lower limb reflexes were slightly increased and one visual evoked response latency was prolonged. His CT and MRI brain scans were normal.

In patient 1 , the neurological disorder may have been due to ceasing the PKU diet or to the marginal vitamin B12 deficiency. His neurological syndrome was not typical of the usual vitamin B12 deficiency state or the neuropsychiatric manifestations of borderline vitamin $\mathrm{B} 12$ deficiency. The patient made a 
significant recovery when he was re-started on the PKU diet and before starting vitamin B12 supplementation. Therefore, while vitamin B12 deficiency should be considered in PKU patients who eat an essentially vegetarian diet, we conclude that, in our patient, the neurological syndrome was probably related to the metabolic abnormalities of PKU.

Untreated PKU causes profound mental retardation and neurological abnormalities and is associated with myelin abnormalities. ${ }^{12}$ These changes may result from the toxic effects of phenylalanine on brain development ${ }^{7}$ and myelin formation. ${ }^{8}$ Treatment of PKU with dietary restriction prevents the development of profound mental retardation although such children may still have a lower IQ than their unaffected siblings. ${ }^{9}$

Some patients with PKU may have intellectual deterioration after ceasing the $\operatorname{diet}^{49}$ but the development of severe neurological abnormalities is not common. In patient 1 there was development of spasticity and abnormalities of the cerebral white matter were present. He also had visual evoked response abnormalities which have previously been found in poorly controlled PKU. ${ }^{10}$ Wood $^{11}$ also reported spasticity in PKU after ceasing dietary restriction and Villasana et $a l^{12}$ reported a PKU patient who developed progressive neurological deterioration which improved after re-instituting dietary restriction. Thompson et $a^{3}$ also reported 7 patients with PKU who developed neurological abnormalities in young adult life. Some of these patients had MRI abnormalities, particularly of the deep posterior white matter, similar to those in our patient.

The histories of these patients and of our patient 1 suggest that, in some patients, dietary relaxation may be followed by the development of neurological signs, particularly spasticity. The observation in our patient that reinstitution of dietary restriction lead to remission of signs without alteration of the MRI findings suggested that the neurological signs were related to the metabolic effects of elevated phenylalanine concentrations. Elevated phenylalanine concentrations influence the metabolism of tyrosine and tryptophan to dopamine and serotonin respectively. ${ }^{1314}$ Phenylalanine can also inhibit glutamic acid decarboxylase which is required for synthesis of the inhibitory neurotransmitter gamma amino butyric acid (GABA). ${ }^{1516} \mathrm{~A}$ failure of synthesis of GABA might have contributed to the spasticity, as spasticity can be treated by GABA agonists.

However, our patient 1 made a substantial recovery even when serum phenylalanine concentrations were still elevated. A possible explanation for this is that the PKU dietary supplement provides a supply of tyrosine.
Tyrosine supplementation in PKU has been found to increase synthesis of neurotransmitters. ${ }^{17}$ Villasana et al ${ }^{12}$ also showed that reinstitution of the PKU diet improved neurotransmitter synthesis in patients with neurological abnormalities.

The following conclusions may be drawn from our two patients and other reports: ${ }^{3} 1112$ Firstly, in some PKU patients, spasticity may develop after ceasing dietary restrictions, but these signs may be reversible; Secondly, even in patients who have been treated since birth and who have minimal mental retardation, abnormalities of the cerebral white matter may develop; Thirdly, these abnormalities may develop in patients homozygous for haplotype 2 PKU (classical PKU) and in our study were not associated with an unusual variant of PKU or with a recognised enzyme deficiency causing leukodystrophy.

We are grateful for the financial assistance of the National Multiple Sclerosis Society and the National Health and Medical Research Council of Australia. Dr P A McCombe holds an NHMRC $R$ Douglas Wright New Investigator Award.

1 Pedersen HE, Birket-Smith E. Neurological abnormalities in phenylketonuria. Acta Neurol Scand 1974;50:589-98.

2 Malamud N. Neuropathology of phenylketonuria. 7 Neuropathol Exp Neurol 1966;25:254-68.

3 Thompson AJ, Smith I, Brenton D, et al. Neurological deterioration in young adults with phenylketonuria. Lancet 1990;336:602-5.

4 Lou HC, Guttler F, Lykkelund C, et al. Decreased vigilance and neurotransmitter synthesis after discontinuation of and neurotransmitter synthesis after discontinuation of dietary treatment for phenylk

5 Di Lella AG, Huang W-M, Woo SLC. Screening for phenylketonuria mutations by DNA amplification with the polymerase chain reaction. Lancet 1988;1:497-9.

6 Guttler F, Ledley FD, Lidsky AS, et al. Correlation between polymorphic DNA haplotypes at phenylalanine hydroxylase locus and clinical phenotypes of phenylketonuria. $f$ Pediatr 1987;110:68-71.

7 Neame KE. Phenylalanine as an inhibitor of transport of amino acids in brain. Nature $1961 ; 192: 173-4$

8 Crome L, Tymms V, Woolf LI. A chemical investigation of the defects of myelination in phenylketonuria. 7 Neurol Neurosurg Psychiat 1962;25:143-8.

9 Williamson ML, Koch R, Azeu C, Chang C. Correlates of intelligence test results in treated phenylketonuric children. Pediatrics 1981;68:161-7.

10 Giovannini M, Valsasina $R$, Villani $R$, et al. Pattern reversal visual evoked potentials in phenylketonuria. $\mathcal{F}$ Inher Metab Dis 1988;11:416-21.

11 Wood B. Neurological disturbance in a phenylketonuric child after discontinuance of dietary treatment. Develop Med Child Neurol 1976;18:657-65.

12 Villasana D, Butler IJ, Williams JC, Roongta SM. Neurological deterioration in adult phenylketonuria. $\mathcal{f}$ Inher Metab Disease 1989;12:451-7.

13 McKean CM. The effect of high phenylalanine concentrations on serotonin and catecholamine metabolism in the human brain. Brain Res 1972;47:469-76.

14 Butler IJ, O'Flynn ME, Seifert WE, Howell RR. Neurotransmitter defects and treatment of disorders of hyperphenylalaninemia. $\mathcal{f}$ Pediatr 1981;98:729-33.

15 Hanson A. Action of phenylalanine metabolites on glutamic acid decarboxylase and $\delta$-aminobutyric acid - $a$-ketoglutaric acid transaminase in brain. Acta Chemica Scandinavitaric acid transaminase

16 Tashian RE. Inhibition of brain glutamic decarboxylase by phenylalanine, valine and leucine derivatives: a suggestion concerning the etiology of the neurological defect in phenylketonuria and branched-chain ketonuria. Meta bolism 1961;10: 393-402.

17 Lykkelund C, Nielsen JB, Lou HC, et al. Increased neurotransmitter biosynthesis in phenylketonuria induced by phenylalanine restriction or by supplementation of unrestricted diet with large amounts of tyrosine. Eur $f$ Pediatr 1988;148:238-45. 